OPEN ACCESS

Edited by:

Pietro Zoppoli,

Oncological Center of Basilicata

(IRCCS), Italy

Reviewed by:

Sabino Russi,

Oncological Center of Basilicata

(IRCCS), Italy

Peng Gao,

Shandong University, China

${ }^{*}$ Correspondence:

Xu-Yong Lin

linxuyong@hotmail.com

Specialty section: This article was submitted to

Cancer Genetics,

a section of the journal

Frontiers in Oncology

Received: 12 September 2019

Accepted: 25 February 2020

Published: 13 March 2020

Citation:

Du J, Liang Y, Li J, Zhao J-M and Lin X-Y (2020) Gastric Cancer Cell-Derived Exosomal microRNA-23a Promotes Angiogenesis by Targeting PTEN. Front. Oncol. 10:326.

doi: 10.3389/fonc.2020.00326

\section{Gastric Cancer Cell-Derived Exosomal microRNA-23a Promotes Angiogenesis by Targeting PTEN}

\author{
Jiang Du ${ }^{1}$, Yuan Liang ${ }^{2}$, Ji Li ${ }^{1}$, Jin-Ming Zhao ${ }^{1}$ and Xu-Yong Lin ${ }^{1 *}$ \\ ${ }^{1}$ Department of Pathology, The First Affiliated Hospital and College of Basic Medical Science, China Medical University, \\ Shenyang, China, ${ }^{2}$ Medical Oncology Department of Thoracic Cancer (2), Cancer Hospital of China Medical University, \\ Liaoning Cancer Hospital \& Institute, Shenyang, China
}

Hypoxia-exposed lung cancer-released exosomal microRNA-23a (miR-23a) has been shown to enhance angiogenesis as well as vascular permeability, contributing to the close correlation between exosomal miR-23a and tumorigenesis. The current study aimed to investigate whether gastric cancer (GC) cell-derived exosomal miR-23a could induce angiogenesis and to elucidate the potential mechanisms associated with the process. Differentially expressed miRNAs in GC were initially screened from the Gene Expression Omnibus database. Target genes were selected following miRNA-mRNA prediction and subsequently verified by dual luciferase reporter assay. RT-qPCR was conducted to detect miR-23a and PTEN expression in GC tissues, cells and exosomes. Human umbilical venous endothelial cells (HUVECs) were co-cultured with GC cell-derived exosomes to assess the angiogenesis mediated by exosomes in vitro. Additionally, PTEN was overexpressed in HUVECs to analyze the mechanism by which miR-23a regulates angiogenesis. miR-23a was highly expressed in GC tissues and cells and GC cell-derived exosomes. Angiogenesis was promoted by the co-culture of HUVECs and GC cells-derived exosomes, as evidenced by the increased expression of VEGF but decreased expression of TSP-1. PTEN was targeted by miR-23a and was lowly expressed in GC tissues. In a co-culture system, miR-23a carried by GC cells-derived exosomes promoted angiogenesis via the repression of PTEN. Collectively, GC cell-derived exosomal miR-23a could promote angiogenesis and provide blood supply for growth of GC cells. This study contributes to advancement of miRNA-targeted therapeutics.

Keywords: exosome, gastric cancer, microRNA-23a, PTEN, AKT pathway, angiogenesis

\section{INTRODUCTION}

Gastric cancer (GC) is a heterogeneous malignancy with epidemiologic and histopathologic differences around the world (1). Global cancer statistics from 2018 revealed that over 1 million people were newly diagnosed with GC resulting in $\sim 0.78$ million deaths (2). At present, surgical intervention remains the only curative approach for GC, while adjuvant and neoadjuvant therapy is usually combined with surgery in locally advanced cases (3). Exosomes have emerged as effective drug and gene therapeutic transporters for a wide variety of pathologies (4). Exosomes are 
cell-derived vesicles with diameter ranging from 20 to $100 \mathrm{~nm}$, capable of influencing angiogenesis, metastasis and other biological cellular properties related to tumorigenesis (5). EGFR delivered by exosomes has been reported to contribute to the liver microenvironment to accelerate liver-specific metastasis of GC (6). Cancer cell-derived exosomes facilitate GC progression by transferring foreign microRNAs (miRNAs), which has shed new light on the mechanism of tumorigenesis and potential novel targets for GC treatment (7). Additionally, genetic and epigenetic abnormalities such as changes in miRNA and gene profiles have been widely implicated in gastric carcinogenesis $(8,9)$.

miRNAs, a group of small non-coding RNA molecules $\sim 19$-25 nucleotides, are dysregulated in GC, and some of them are related with GC progression and prognosis (10). miRNAs are important mediators of gene expression that guide their inhibition via pairing to messenger RNAs (mRNAs) of protein-coding genes (11). Additionally, miRNAs can typically inhibit the genes participated in the regulation of cellular processes including inflammation, cell cycle progression, stress response, cell differentiation and apoptosis (12). miRNAs control those cellular processes by regulation of oncogenes or antioncogenes, thereby affecting occurrence of gastric cancer (13). More importantly, it was documented that miRNAs play pivotal roles in mediation of gastric cancer stem cells and GC EMT $(14,15)$. For example, miR-375 functions as a tumor inhibitor to potentially modulate the growth of GC cells by targeting the oncogene JAK2 (16). A previous study revealed that miR$23 \mathrm{a} / \mathrm{b}$ targets PDCD4 to induce tumorigenesis and to inhibit apoptosis of GC cells, suggesting an oncogenic role of miR$23 \mathrm{a} / \mathrm{b}$ (17). Additionally, the downregulation of miR-23a has been shown to negatively regulate the tumor suppressor, phosphatase and tensin homologue (PTEN), to enhance the sensitivity of lung cancer stem cells to erotinib (18). Interestingly, the loss of PTEN facilitates cancer cell proliferation and contributes to tumorigenesis (19). In this regard, we speculated that exosomal miR-23a might target PTEN to contribute to gastric carcinogenesis. Based on previous researches, the current study was designed to investigate whether cancer cell-derived exosomes can carry miR-23a to promote the development of GC with the aim of identifying new therapeutic strategies as well as the underlying molecular mechanisms associated with PTEN.

\section{MATERIALS AND METHODS}

\section{Ethics Statement}

The experiment was approved by the Ethics Committee of the First Affiliated Hospital of China Medical University and performed in strict accordance with the Declaration of Helsinki. All patients signed informed written consent documents.

\section{Microarray-Based Analysis}

miRNA microarray data related to GC were retrieved from the Gene Expression Omnibus (GEO) database (https://www. ncbi.nlm.nih.gov/geo/), followed by differential analysis using the Limma package of $\mathrm{R}$ language. Next, a heatmap of the differentially expressed miRNAs was plotted using the pheatmap package. Expression of miR-23a in GC samples provided by the
Cancer Genome Atlas (TCGA) was analyzed by starBase database (http://starbase.sysu.edu.cn/panCancer.php). The possible target genes of miR-23a were obtained using miRDB (http://starbase. sysu.edu.cn/panCancer.php), mirDIP (http://ophid.utoronto. ca/mirDIP/index.jsp\#r), starBase (http://starbase.sysu.edu.cn/ agoClipRNA.php? source $=$ mRNA), and TargetScan (http://www. targetscan.org/vert_71/). MalaCards database (https://www. malacards.org/) was used to search the known GC-related genes, and subsequently STRING database (https://string-db.org/) was used to construct a gene interaction network map.

\section{Study Subjects}

Forty GC patients who underwent GC resection at the First Affiliated Hospital of China Medical University from June 2018 to November 2018 were enrolled into the study. GC and adjacent normal tissues were collected from the participating patients. Among the enrolled patients, 22 cases were confirmed to be positive for hydrophobic-hydrophilic $\left(\mathrm{HP}^{+}\right)$and 18 were confirmed to be negative for $\mathrm{HP}\left(\mathrm{HP}^{-}\right)$. All tissue specimens were confirmed to be GC tissue by pathological examination, with none of the participants yet to receive local or systemic antitumor treatment prior to surgery. Patients who died from a nonGC or accident were excluded from the study. Following surgery, all tissue specimens were quickly frozen in liquid nitrogen for $1 \mathrm{~h}$ after being rinsed with normal saline, and stored at $-80^{\circ} \mathrm{C}$.

\section{Cell Treatment}

The GC cell lines NCI-N87, HGC-27, AGS (Cell Bank of China Center for Type Culture Collection, Shanghai, China), MKN45 (CC-Y1358), and normal gastric mucosal epithelial cell line GES-1 (CC-Y1572) (EK-Bioscience, Shanghai, China) were subjected to mycoplasma test and short tandem repeat. The cells were cultured with Dulbecco's Modified Eagle's Medium (DMEM; Thermo Fisher Scientific Inc., Waltham, MA, USA) supplemented with $10 \%$ fetal bovine serum (FBS; Thermo Fisher Scientific Inc., Waltham, MA, USA.), $100 \mathrm{U} / \mathrm{mL}$ penicillin, and $100 \mu \mathrm{g} / \mathrm{mL}$ streptomycin.

The cells at logarithmic growth phase were seeded in a 6-well plate $\left(6.0 \times 10^{5}\right.$ cells/well $)$, and then transfected with negative control (NC)-mimic, miR-23a mimic, NC-inhibitor and miR-23a inhibitor as outlined in the instructions of Lipofectamine 2000 kit (Invitrogen, Carlsbad, CA, USA). After the transfected cells were cultured for $48 \mathrm{~h}$, the exosomes were subsequently extracted.

\section{Extraction of Exosomes (20)}

GC cell-derived exosomes were extracted by differential centrifugation. All centrifugation steps were performed at $4^{\circ} \mathrm{C}$, and other steps were performed on ice. In short, cell debris and dead cells were removed following centrifugation at $500 \mathrm{~g}$ for $10 \mathrm{~min}$ and at 2,000 g for $20 \mathrm{~min}$ in a cryogenic centrifuge (Eppendorf, Germany). The supernatant was centrifuged at $110,000 \mathrm{~g}$ for $45 \mathrm{~min}$ in a high-speed centrifuge (Beckman, USA) to remove cell debris, large cells and extracellular vesicles. The supernatant was filtered using a $0.22 \mu \mathrm{m}$ filter. Next, the exosomes were isolated at $110,000 \mathrm{~g}$ for $90 \mathrm{~min}$ in an ultracentrifuge (Beckman, USA). The collected pellets were resuspended in phosphate buffered saline (PBS) and repeatedly 
centrifuged once. Finally, the pellets were resuspended in 50-100 $\mu \mathrm{L}$ PBS and stored at $-80^{\circ} \mathrm{C}$ for subsequent use. The exosome markers TSG101, CD63 and Alix were measured by Western blot analysis.

\section{Transmission Electron Microscope (TEM)}

Firstly, the separated exosomes were thoroughly mixed with PBS. The sample was adsorbed on a carbon-coated nickel grid and counter-stained with $2 \%$ methyl tungstate for $5 \mathrm{~min}$. The stain was then wiped off from the grid using tinder paper. After being dried, the samples were observed under a JEM-1230 electron microscope (Nihon Denshi, Tokyo, Japan) at an acceleration voltage of $80 \mathrm{kV}$.

\section{Nanoparticle Size Analysis}

Firstly, the obtained exosome precipitate following centrifugation was suspended in $500 \mu \mathrm{L}$ PBS at a ratio of 1:100 and stored at $-20^{\circ} \mathrm{C}$. Nanoparticle size measurement was then performed using a Nanosight LM10-HS nanoparticle analyzer (Great Malvern, UK).

\section{Reverse Transcription Quantitative Polymerase Chain Reaction (RT-qPCR)}

The total RNA of the tissues or cells was extracted in strict accordance with the instructions of the TRIZOL kit (15596-018, Beijing solarbio science \& technology Co., Ltd., Beijing, China), after which the concentration of RNA was determined. The obtained RNA was then subjected to reverse transcription using a miRNA reverse transcription kit (D1801, Harbin Xinhai Gene Co., Ltd., Harbin, China) and complementary DNA (cDNA) reverse transcription kit (K1622, Beijing Yaanda Biotechnology Co., Ltd., Beijing, China). Next, qPCR was performed on a ViiA 7 Real-Time PCR System (Daan Gene Co., Ltd., of Sun Yatsen University, Guangzhou, China) with $2 \mu \mathrm{g}$ total cDNA as template. U6 and GAPDH were used as internal references. The relative transcription levels of the target genes were calculated using the $2{ }^{-\Delta \Delta \mathrm{Ct}}$ method (21). The primers used in this study were synthesized by Takara (Dalian, China) (Table 1).

TABLE 1 | Primer sequences for RT-qPCR.

\begin{tabular}{ll}
\hline Gene & Primer sequence \\
\hline miR-23a & F: AGCAGCCAGTTACCCAAGA \\
& R: TGACAGTGCGAGACTCCATC \\
PTEN & F: GGACGAACTGGTGTAATGATATG \\
& R: TCTACTGTIITGTGAAGTACAGC \\
VEGF & F: CACATAGGAGAGATGAGCTTC \\
& R: CCGCCTCGGCTTGTCACAT \\
U6 & F: CTCGCTCGGCAGCACA \\
& R: AACGCTTCACGAATTGCGT \\
GAPDH & F: GAAGGTGAAGGTCGGAGTCA \\
& R: AATGAAGGGGTCATTGATGG
\end{tabular}

$R T$-qPCR, reverse transcription quantitative polymerase chain reaction; $F$, forward; $R$, reverse; miR-23a, microRNA-23a; PTEN, Phosphatase and tensin homologue deleted on chromosome 10; VEGF, vascular endothelial growth factor; GAPDH, glyceraldehyde3-phosphate dehydrogenase.

\section{Western Blot Analysis}

Total protein was extracted by lysis for $15 \mathrm{~min}$ at $4^{\circ} \mathrm{C}$ and centrifugation at $15,000 \times \mathrm{g}$ for $15 \mathrm{~min}$. Next, the supernatant was extracted, and the protein concentration was determined using a bicinchoninic acid kit (20201 ES76, Yeasen Biotechnology Co., Ltd., Shanghai, China). The protein was then separated by electrophoresis and then transferred onto a polyvinylidene fluoride membrane. The membrane was blocked with 5\% bovine serum albumin for $1 \mathrm{~h}$ and incubated overnight at $4^{\circ} \mathrm{C}$ with the following primary antibodies: rabbit anti-human PTEN (1:10,000, ab32199, Abcam Inc., Cambridge, UK), mouse anti-human vascular endothelial growth factor (VEGF; 1:1,000, AV202-1, Beyotime Institute of Biotechnology, China), thrombospondin-1 (TSP-1; 1:10,000, ab85762, Abcam Inc., Cambridge, UK) and glyceraldehyde 3-phosphate dehydrogenase (GAPDH; 1:10,000, ab181602, Abcam Inc., Cambridge, UK). The membrane was then incubated with the horseradish peroxidaselabeled goat anti-rabbit immunoglobulin G (ab205718, 1:20,000, Abcam Inc., Cambridge, UK) for $1 \mathrm{~h}$. The bands were developed using enhanced chemiluminescence and quantified using ImageJ $1.48 \mathrm{u}$ software (Wayne Rasband, National Institutes of Health, Bethesda, MD, USA). The ratio of the gray value of target proteins band to GAPDH band was expressed as the relative protein expression.

\section{Co-culture of Human Umbilical Venous Endothelial Cells (HUVECs) and GC Cells-Derived Exosomes}

The exosomes were isolated from the HGC-27 cells transfected miR-23a inhibitor, NC-inhibitor, miR-23a mimic or NC-mimic (exo-miR-23a inhibitor, exo-NC-inhibitor, exo-miR-23a mimic, or exo-NC-mimic). The PBS-suspended exosomes were mixed with carboxy fluorescein succinimidyl ester (CFSE) (C1031, Beyotime Institute of Biotechnology, Shanghai, China) at a volume ratio of $10: 1$, and incubated at $37^{\circ} \mathrm{C}$ for $10 \mathrm{~min}$, which was then terminated with $100 \mu \mathrm{L}$ stop buffer followed by incubation at $4{ }^{\circ} \mathrm{C}$ for $30 \mathrm{~min}$. After 3 -min centrifugation at $14,000 \mathrm{rpm}$, the fluorescence-labeled exosomes resuspended in $200 \mu \mathrm{L}$ PBS were then mixed with exosome-free medium. The exosomes were co-cultured with the 50-60\% confluent HUVECs. HUVECs were incubated only with PBS as control. Meanwhile, exomiR-23a mimic was co-cultured with HUVECs transfected with overexpressed (oe)-PTEN or oe-NC. After incubation in 24well plates for $48 \mathrm{~h}$, the nuclei of the HUVECs were stained with 4,6-diamidino-2-phenylindole (DAPI, Abbott, USA) and subsequently observed under a confocal microscope (FV10i, Olympus Optical Co., Ltd, Tokyo, Japan) to determine the internalization of exosomes derived from the GC cells. Finally, the expression of miR-23a was quantified by RT-qPCR.

\section{5-Ethynyl-2' -Deoxyuridine (EdU) Assay}

The cells were seeded at a density of $5 \times 10^{4}$ cells $/ \mathrm{mL}$. Based on the instructions of EdU kit (C10310, Ribobio, Guangzhou, China), the cells were incubated with EdU solution (100 $\mathrm{LL} /$ well $)$ for $2 \mathrm{~h}$, and fixed with cell fixative $(100 \mu \mathrm{L} /$ well $)$ for $30 \mathrm{~min}$ at ambient temperature. The cells were incubated with $2 \mathrm{mg} / \mathrm{mL}$ 
glycine for $5 \mathrm{~min}$, and permeabilized with PBS containing $0.5 \%$ TritonX-100 (100 $\mu \mathrm{L} /$ well $)$ for $10 \mathrm{~min}$. The cells were then incubated with $1 \times$ Apollo reaction solution under conditions void of light for $30 \mathrm{~min}$, and permeabilized with PBS containing $0.5 \%$ TritonX-100. The cells were then mounted with antifade mounting medium containing Hoechst. Image capture was performed under a fluorescence microscope, with the number of cells labeled with EdU recorded from three arbitrarily selected fields. The cells with nucleus stained in red were regarded as positive. EdU labeling rate $(\%)=$ the number of positive cells/(the number of positive cells + the number of negative cells $) \times 100 \%$.

\section{In vitro Angiogenesis Assay}

HUVECs (Catalog \#5000, ScienCell Research Laboratories, Carlsbad, CA, USA) were cultured in endothelial cell growth medium (EGM-2) containing 10\% FBS. Next, $20 \mu \mathrm{L}$ BD Matrigel $^{\mathrm{TM}}$ Matrix was diluted using serum-free RPMI-1640 medium (total amount of $40 \mu \mathrm{L}$ ) at a ratio of $1: 1$, which was then added into the upper surface of polyester film in the Transwell chamber (membrane well size of $8 \mu \mathrm{m}$ ) and dried in a fume hood at room temperature for $1 \mathrm{~h}$. Subsequently, $200 \mu \mathrm{L}$ cell suspension $\left(2 \times 10^{5}\right.$ cells $\left./ \mathrm{mL}\right)$ was loaded to the apical chamber. The exosomes were then placed into the basolateral chamber. After $24 \mathrm{~h}$ of incubation in a $37^{\circ} \mathrm{C}$ wet incubator with $5 \% \mathrm{CO}_{2}$, the branch nodes of the pseudo-tube-like and end-to-end tubular structure formed in HUVECs were observed in five randomly selected visual fields and numbered under an optical microscope.

\section{Dual Luciferase Reporter Gene Assay}

The relationship between miR-23a and PTEN was predicted in the Targetscan website (http://www.targetscan.org), and further verified using dual luciferase reporter gene assay. The plasmids named PTEN-wild type (Wt) and PTEN-mutant (Mut) were constructed, after which the two plasmids were co-transfected

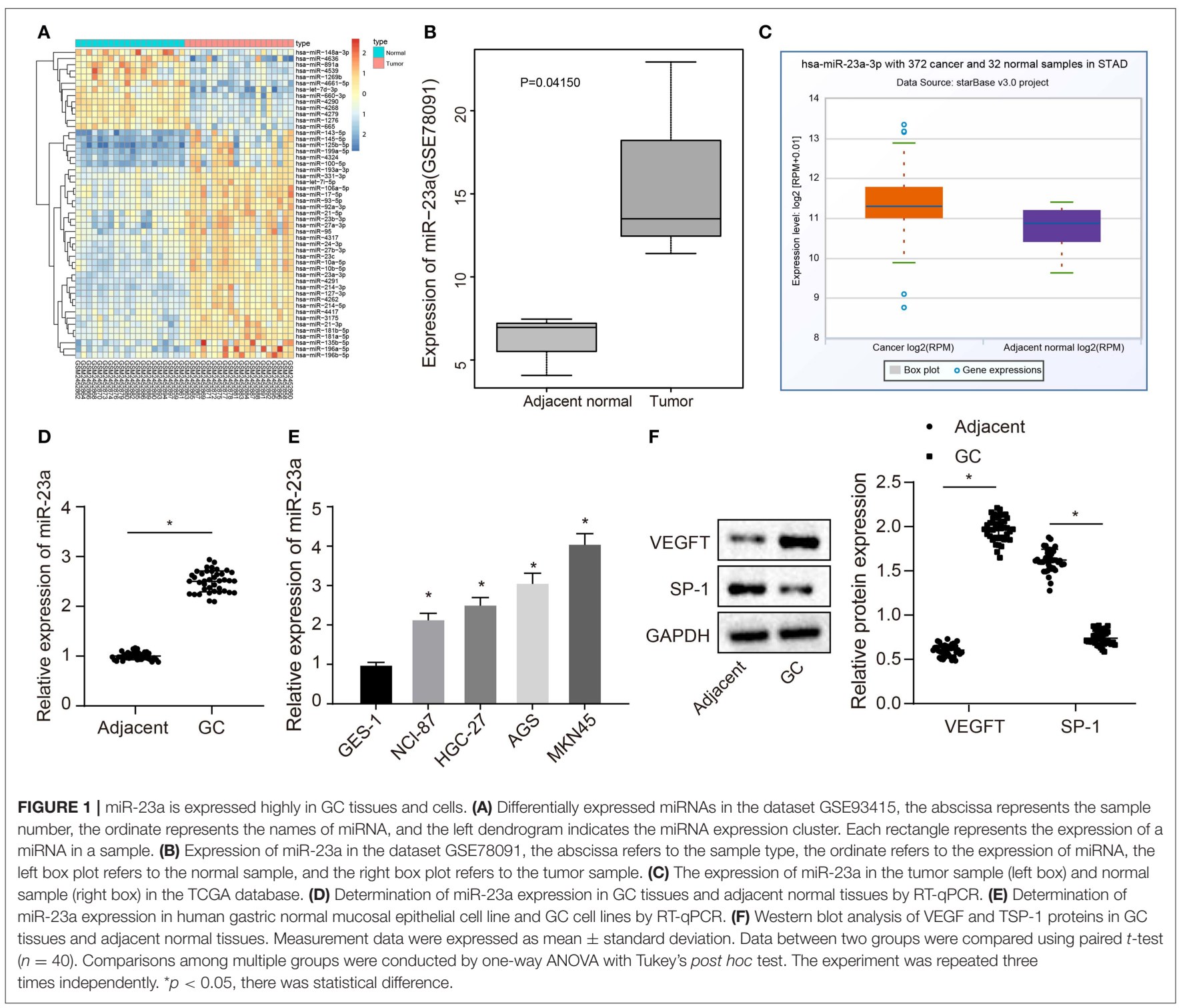


into HEK-293T cells with miR-23a mimic and NC-mimic, respectively. After transfection for $24 \mathrm{~h}$, the cells were lysed and centrifuged at 12,000 rpm for $1 \mathrm{~min}$, followed by collection of the supernatant. Luciferase activity was then detected using the Dualluciferase ${ }^{\circledR}$ Reporter Assay System (E1910, Promega, Madison, WI, USA).

\section{Statistical Analysis}

All statistical analyses were conducted using SPSS 21.0 statistical software (IBM Corp. Armonk, NY, USA). Measurement data were expressed as mean \pm standard deviation. If the data were in compliance with normal distribution and homogeneity of variance, paired data between two groups were compared using paired t test, and unpaired data between two groups were compared between using unpaired $t$-test. Comparisons among multiple groups were evaluated by oneway analysis of variance (ANOVA) with Tukey's post-hoc test. A value of $p<0.05$ was considered to be indicative of statistical difference.

\section{RESULTS}

\section{High Expression of miR-23a Is Detected in GC Tissues and Cells}

The GC-associated miRNA expression dataset GSE93415 was obtained through retrieval from the GEO database. The results indicated 76 differently expressed miRNAs in GC. The heatmap depicted the top 50 miRNAs exhibiting larger fold changes (Figure 1A). Among them, miR-23a was the miRNA with the largest fold change. Meanwhile, based on the GSE78091 dataset retrieved from the GEO database and data from TCGA, we detected that miR-23a was robustly induced in GC (Figures 1B,C). High expression of miR-23a in GC was further demonstrated by RT-qPCR. As illustrated in Figure 1D, compared with adjacent normal tissues, miR-23a expression was appreciably increased in GC tissues. Further correlation analysis exhibited that miR-23a expression was positively with tumor node metastasis (TNM) and not correlated with age, histological grade, and gender (Table 2). Subsequently, RT-qPCR data displayed remarkably higher miR-23a expression in GC cell lines NCI-N87, HGC-27, AGS, and MKN45 than in normal mucosal epithelial cell line GES-1 (Figure 1E). These results confirmed that miR-23a was highly expressed in GC tissues and cells. In addition, Western blot analysis exhibited that compared with adjacent normal tissues, the protein expression of VEGF was notably increased, but that of TSP-1 was diminished in GC tissues (Figure 1F), indicating that tube formation was more prominent in GC tissues.

\section{miR-23a Is Enriched in GC Cells-Derived Exosomes}

Studies have shown that miR-23a can be carried by tumor cell-secreted exosomes such as nasopharyngeal carcinoma or lung cancer cells and then exert their regulatory functions on angiogenesis (22-24), which suggested that miR-23a carried by GC cell-derived exosomes might be involved in angiogenesis.
TABLE 2 | Correlation between miR-23a expression and clinicopathological characteristics.

\begin{tabular}{|c|c|c|c|c|c|c|}
\hline & \multirow[t]{2}{*}{ Variables } & \multicolumn{2}{|c|}{ Expression } & \multirow[t]{2}{*}{ Total } & \multirow[t]{2}{*}{$x^{2}$} & \multirow[t]{2}{*}{$p$-value } \\
\hline & & Low & High & & & \\
\hline \multirow[t]{3}{*}{ Age (year) } & & & & & 0.44 & 0.507 \\
\hline & $\leq 60$ & 12 & 14 & 26 & & \\
\hline & $>60$ & 8 & 6 & 14 & & \\
\hline \multirow[t]{3}{*}{ Histological grade } & & & & & 2.667 & 0.103 \\
\hline & Low & 10 & 15 & 25 & & \\
\hline & High & 10 & 5 & 15 & & \\
\hline \multirow[t]{3}{*}{ Sex } & & & & & 2.506 & 0.113 \\
\hline & Female & 7 & 12 & 19 & & \\
\hline & Male & 13 & 8 & 21 & & \\
\hline \multirow[t]{3}{*}{ TNM stage } & & & & & 17.29 & $<0.001$ \\
\hline & $1 / I I$ & 18 & 5 & 23 & & \\
\hline & III/IV & 2 & 15 & 17 & & \\
\hline \multirow[t]{3}{*}{ N stage } & & & & & 10.16 & 0.001 \\
\hline & NO & 19 & 10 & 29 & & \\
\hline & N1/N2/N3 & 1 & 10 & 11 & & \\
\hline \multirow[t]{3}{*}{ M stage } & & & & & 8.64 & 0.003 \\
\hline & MO & 17 & 8 & 25 & & \\
\hline & M1 & 3 & 12 & 15 & & \\
\hline
\end{tabular}

To validate this hypothesis, exosomes were extracted from GES1, NCI-N87, HGC-27, AGS, and MKN45 using the ExoQuick method and then identified. The ultrastructure of exosomes was observed under the TEM. It can be clearly observed that the extracts exhibited typical morphological features of exosomes, varying in diameter, with a spherical structure formed by a lipid bilayer molecular membrane. The outer layer of two-layer lipid molecular membrane was deeply dyed, and the interior was heterogeneously light-stained, where protein density substance was visible (Figure 2A). Subsequent results of nanoparticle size analysis (Figure 2B) displayed that the diameter of extracted exosomes was around $100 \mathrm{~nm}$. Next, expression of exosome surface markers (CD63, Alix, and TSG101) in GC cells was highly expressed in exosomes (Figure 2C). RT-qPCR was used to measure the expression of miR-23a in exosomes derived from normal gastric cell line GES-1 and GC cell lines. As depicted in Figure 2D, compared with GES-1 cell-derived exosomes, miR23a expression in GC cell-derived exosomes was significantly increased, and HGC-27 cell-derived exosomes exhibited the highest expression of miR-23a. Therefore, HGC-27 cells were chosen for the subsequent experiments. The aforementioned results demonstrated the enrichment of miR-23a in the exosome derived from GC cells.

\section{Co-culture of HUVECs and GC Cell-Derived Exosomes Stimulates Angiogenesis}

In order to clarify whether co-culture of HUVECs and GC cell-derived exosomes could promote angiogenesis, HUVECs were co-cultured with HGC-27-derived exosomes or PBS. CFSE was used to label exosomes (green) and DAPI to label nuclei of HUVECs (blue), and then the exosome internalization of 


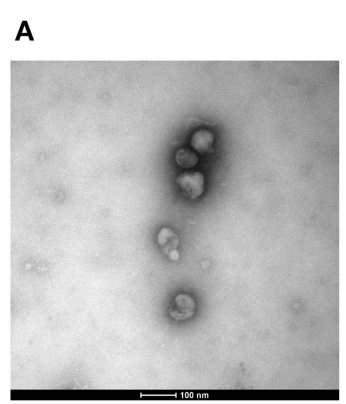

C

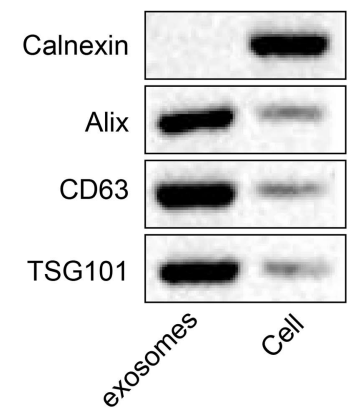

B
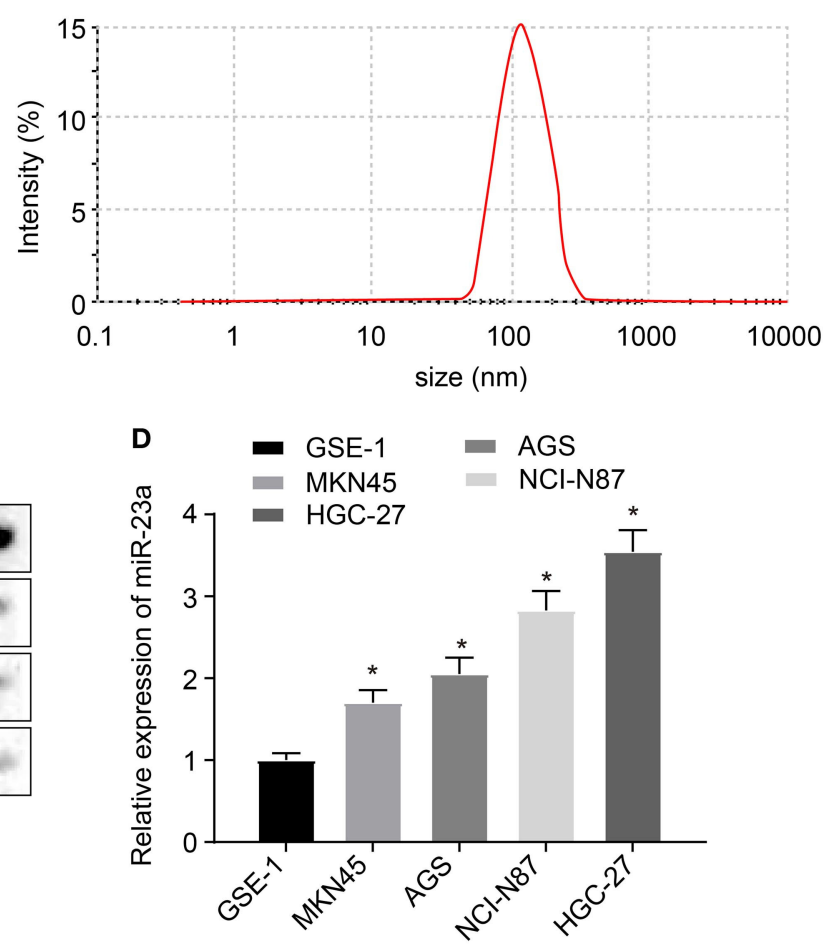

FIGURE 2 | miR-23a exists in the exosomes derived from GC cells. (A) Observation of exosome morphology by transmission electron microscope (scale bar = $100 \mathrm{~nm}$ ), red arrows refer to exosome. (B) Nanoparticle size analysis of exosomes. (C) Western blot analysis of TSG101, CD63 and Alix proteins in isolated exosomes. (D) Determination of miR-23a expression in exosomes isolated from normal gastric cell lines and GC cell lines by RT-qPCR. * $p<0.05$ vs. GSE-1 cell line. Measurement data were expressed as mean \pm standard deviation. Comparisons among multiple groups were conducted by one-way ANOVA with Tukey's post hoc test. The experiment was repeated three times independently.

HUVECs was observed under the confocal microscope. More CFSE-exosomes were internalized by HUVECs with the time prolonged (Figure 3A). As depicted in Figures 3B-E, tube formation ability of HUVECs co-cultured with HGC-27-derived exosomes was enhanced, evidenced by increased tube length and number of loops and nodes. In addition, expression of VEGF and TSP-1 upon co-culture of HUVECs with HGC-27-derived exosomes was measured by Western blot analysis. The results (Figures 3F,G) presented that the protein expression of VEGF upon co-culture of HUVECs with HGC-27-derived exosomes was notably increased, while that of TSP-1 was reduced, which further confirmed that exosomes from GC cells can facilitate the tube formation ability. EdU assay consistently documented (Figure $3 \mathrm{H}$ ) that the proliferation of HUVECs co-cultured with HGC-27-derived exosomes was markedly increased. Based on the above results, it can be concluded that exosomes derived from GC cells can promote angiogenesis and proliferation of HUVECs.

\section{Transfer of miR-23a via GC Cell-Derived Exosomes Promotes Angiogenesis}

To further elucidate the effects of GC cell-derived exosomes on tube formation via miR-23a, HGC-27 cells were transfected with miR-23a mimic or inhibitor to alter endogenous miR-23a level. RT-qPCR revealed that miR-23a expression was upregulated in exosomes derived from HGC-27 cells that had been transfected with miR-23a mimic, whereas that was decreased in exosomes derived from miR-23a inhibitor-transfected HGC27 cells (Figure 4A). As illustrated in Figures 4B-E, the tube formation ability of HUVECs co-cultured with exosomes derived from miR-23a mimic-transfected HGC-27 cells was enhanced, and the tube length and number of loops and nodes were potently increased. Conversely, the tube formation ability of HUVECs co-cultured with exo-miR-23a inhibitor was inhibited. Western blot analysis subsequently manifested that the protein expression of VEGF was strikingly elevated while that of TSP-1 was diminished in HUVECs co-cultured with exosomes derived from miR-23a mimic-transfected HGC-27 cells (Figures 4F,G). On the contrary, the protein expression of VEGF was significantly reduced while that of TSP-1 was elevated in HUVECs co-cultured with exosomes derived from miR23a inhibitor-transfected HGC-27 cells. The EdU assay results (Figures 4H,I) displayed that the proliferation of HUVECs co-cultured with exosomes derived from miR-23a mimictransfected HGC-27 cells was significantly increased, but that of HUVECs co-cultured with exosomes derived from miR-23a inhibitor-transfected HGC-27 cells was obviously reduced. Taken together, exosomal miR-23a secreted from GC cells can promote tube formation. 


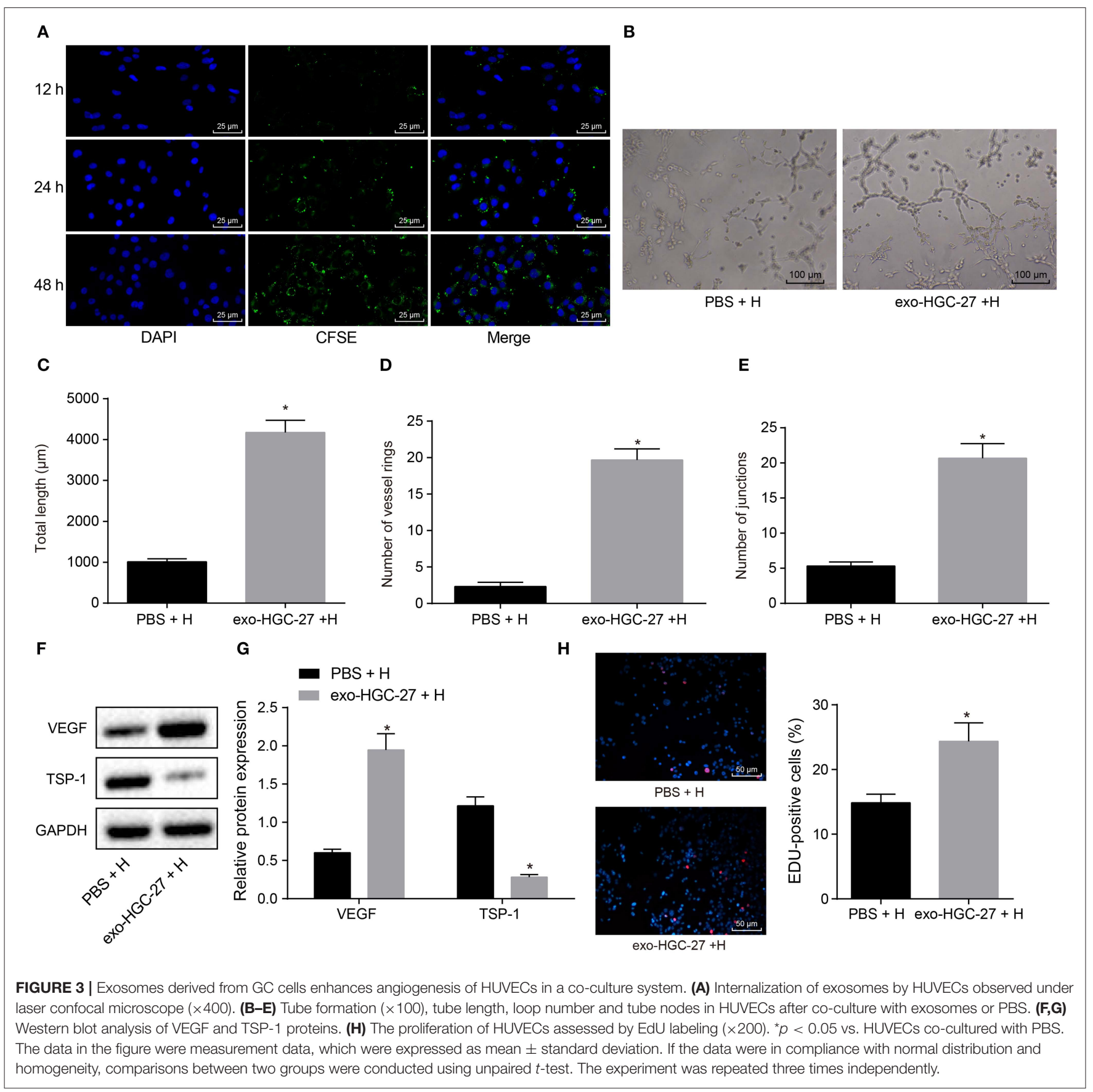

\section{PTEN Is a Target Gene of miR-23a}

To further understand the mechanism of miR-23a in GC, miRDB, mirDIP, starBase and TargetScan databases were used to predict the downstream genes of miR-23a. To improve the prediction accuracy, the target genes with the scores higher than 70 points predicted by miRDB and those with the scores higher than 0.7 points in mirDIP along with the top 500 target genes in the remaining two databases were intersected, and 28 overlapping potential target genes were finally obtained (Figure 5A). Simultaneously, the MalaCards database was adopted to retrieve the most likely genes involved in GC development from these 28 potential target genes, which predicted 10 known genes related to GC. Next, correlation analysis was conducted for the GC-related known genes and the potential target genes of miR-23a. According to the gene interaction network map, the PTEN gene was located at the core (Figure 5B). Next, TargetScan database (http://www.targetscan.org) predicted a binding site between miR-23a and PTEN (Figure 5C). In order to confirm their relationship, dual-luciferase reporter gene assay was performed. As depicted in Figure 5D, the luciferase 


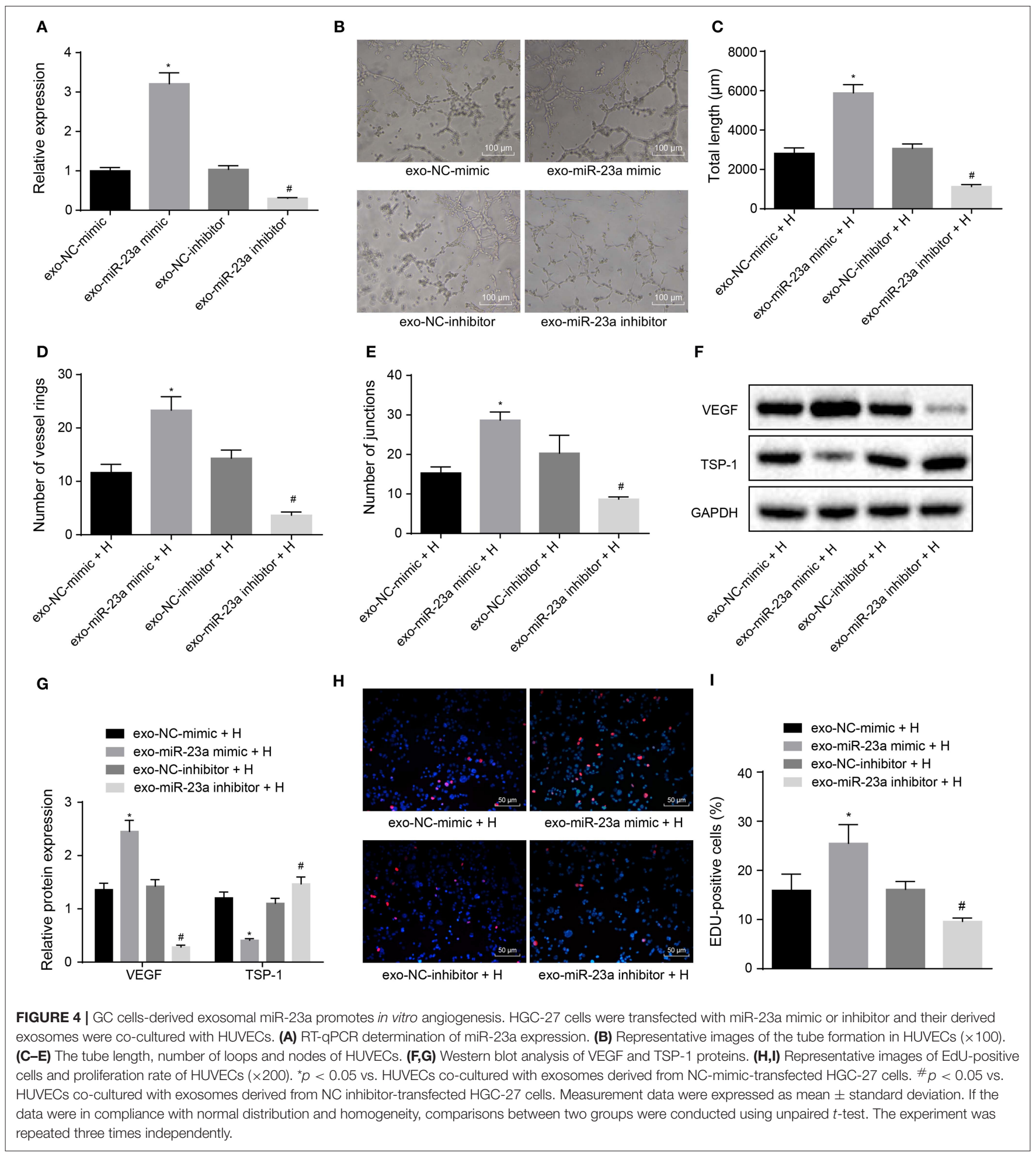

activity of PTEN-3'-UTR-Wt was significantly inhibited by miR-23a mimic, while that of PTEN-3'-UTR-Mut remained unaffected. Moreover, RT-qPCR revealed that PTEN expression was remarkably lower in GC tissues than in adjacent normal tissues (Figure 5E). Through correlation analysis, it was observed that there was inverse correlation between miR-23a and PTEN expression (Figure 5F). Further, PTEN expression was negatively with TNM, and had no significant relationship to age, histological 
A

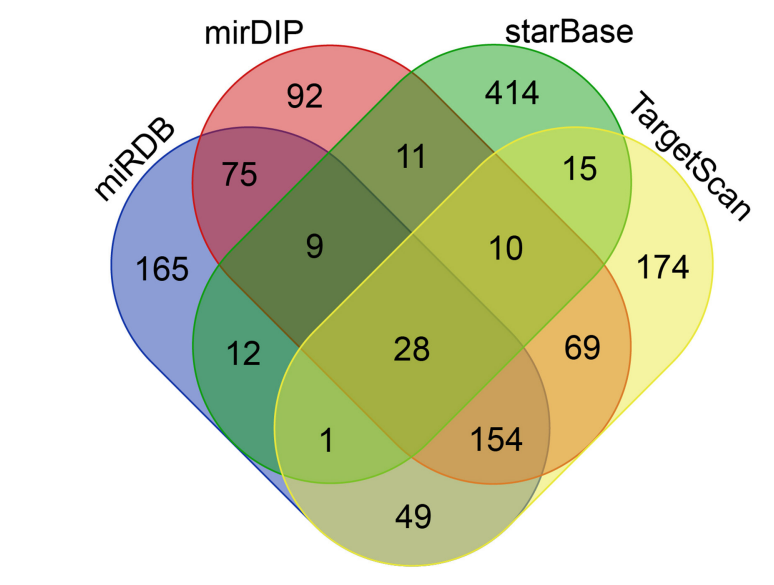

B

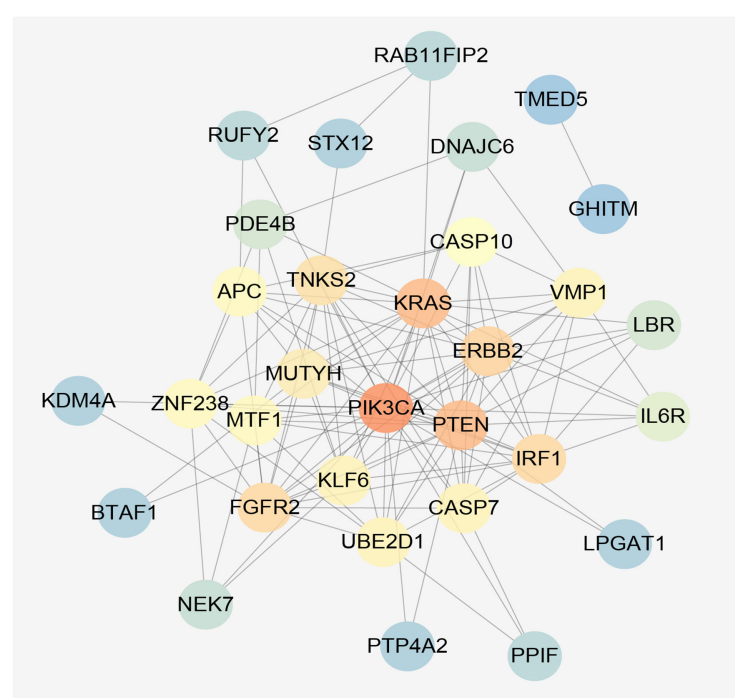

C

\begin{tabular}{|c|c|c|c|c|c|c|c|}
\hline & $\begin{array}{l}\text { Predicted consequential pairing of target region (top) } \\
\text { and miRNA(bottom) }\end{array}$ & $\begin{array}{l}\text { Site } \\
\text { type }\end{array}$ & $\begin{array}{c}\text { Context++ } \\
\text { score }\end{array}$ & $\begin{array}{c}\text { Context++ } \\
\text { score percentile }\end{array}$ & $\begin{array}{c}\text { Weighted } \\
\text { Context++ score }\end{array}$ & $\begin{array}{c}\text { Conserved branch } \\
\text { length }\end{array}$ & PCT \\
\hline $\begin{array}{l}\text { Position 3383-3390 of PTEN 3' UTR } \\
\text { hsa-miR-23a-3p }\end{array}$ & 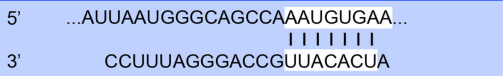 & $8 \mathrm{mer}$ & -0.09 & 76 & -0.06 & 0.313 & $<0.1$ \\
\hline
\end{tabular}

D

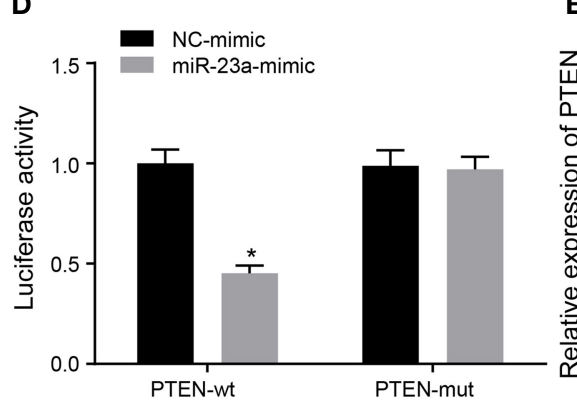

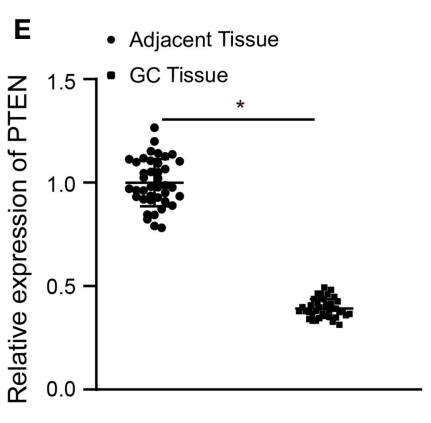

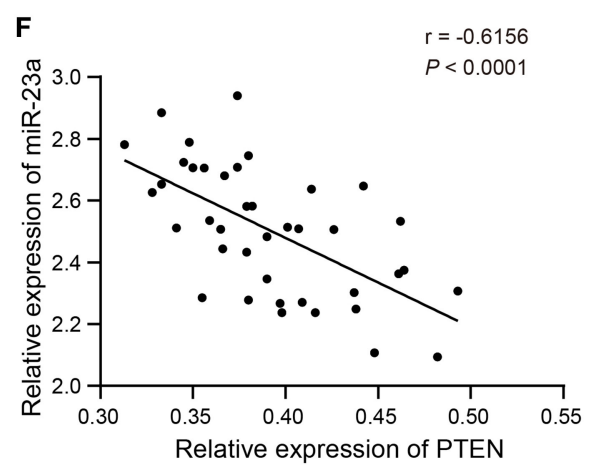

FIGURE 5 | miR-23a targets PTEN and negatively regulated its expression. (A) Intersection of predicted target genes of miR-23a based on the results of four databases, where the middle part represents the intersection. (B) Correlation analysis of the known genes related to GC with target genes of miR-23a. Each small circle in the figure represents a gene, and the line between the circles indicates the interaction between two genes. (C) The predicted binding sites of miR-23a on the PTEN by TargetScan website. (D) The binding relationship between miR-23a and PTEN confirmed by dual-luciferase reporter gene assay. (E) PTEN expression in GC tissues and adjacent normal tissues detected by RT-qPCR. (F) Correlation between miR-23a and PTEN expression analyzed by Pearson. ${ }^{*} p<0.05$ vs. cells transfected with NC-mimic. Measurement data were expressed as mean \pm standard deviation. If the data were in compliance with normal distribution and homogeneity, comparisons between two groups were conducted using unpaired $t$-test. The experiment was repeated three times independently.

grade and gender (Table 3). Therefore, miR-23a directly targeted PTEN.

\section{Exosomal miR-23a Promotes Angiogenesis and Activates the AKT Pathway by Inhibiting PTEN}

To verify that miR-23a inhibits PTEN expression to promote angiogenesis, oe-PTEN-transfected HUVECs were co-cultured with the exosomes derived from miR-23a mimic-transfected HGC-27 cells. RT-qPCR (Figure 6A) and Western blot analysis (Figures 6B,C) provided data showing that mRNA and protein expression of TSP-1 was increased substantially in oe-PTEN-transfected HUVECs co-cultured with exosomes-miR-23a mimic, but that of PIP3 and phosphorylated Akt/Akt was reduced as compared to the oe-NC transfected HUVECs co-cultured with exosomesmiR-23a mimic. Subsequent results from tube formation experiment (Figures 6D-G) showed that the ability of tube formation was weakened in oe-PTEN-transfected HUVECs co-cultured with exosomes-miR-23a mimic, which was indicated by diminished tube length and number of loops and nodes, in contrast to oe-NC transfected HUVECs co-cultured with exosomes-miR-23a mimic. At the same time, Western blot analysis (Figures $\mathbf{6 H}, \mathbf{I}$ ) showed that the protein expression of TSP-1 was significantly increased in oe-PTEN-transfected 
TABLE 3 | Correlation between miR-23a expression and clinicopathological characteristics.

\begin{tabular}{|c|c|c|c|c|c|c|}
\hline & \multirow[t]{2}{*}{ Variables } & \multicolumn{2}{|c|}{ Expression } & \multirow[t]{2}{*}{ Total } & \multirow[t]{2}{*}{$x^{2}$} & \multirow[t]{2}{*}{$p$-value } \\
\hline & & Low & High & & & \\
\hline \multirow[t]{3}{*}{ Age (year) } & & & & & 1.192 & 0.275 \\
\hline & $\leq 60$ & 11 & 15 & 26 & & \\
\hline & $>60$ & 9 & 6 & 14 & & \\
\hline \multirow[t]{3}{*}{ Histological grade } & & & & & 0.007 & 0.936 \\
\hline & Low & 14 & 11 & 25 & & \\
\hline & High & 6 & 5 & 15 & & \\
\hline \multirow[t]{3}{*}{ Sex } & & & & & 0.742 & 0.342 \\
\hline & Female & 11 & 8 & 19 & & \\
\hline & Male & 9 & 12 & 21 & & \\
\hline \multirow[t]{3}{*}{ TNM stage } & & & & & 12.38 & 0.0004 \\
\hline & $\mid / I I$ & 6 & 17 & 23 & & \\
\hline & III/IV & 14 & 3 & 17 & & \\
\hline \multirow[t]{3}{*}{ N stage } & & & & & 6.144 & 0.013 \\
\hline & NO & 11 & 18 & 29 & & \\
\hline & N1/N2/N3 & 12 & 8 & 11 & & \\
\hline \multirow[t]{3}{*}{ M stage } & & & & & 5.227 & 0.022 \\
\hline & MO & 9 & 16 & 25 & & \\
\hline & M1 & 11 & 4 & 15 & & \\
\hline
\end{tabular}

HUVECs co-cultured with exosomes-miR-23a mimic, but that of VEGF was significantly reduced in contrast to oe-NC transfected HUVECs co-cultured with exosomes-miR-23a mimic. EdU assay results suggested that the proliferation of oe-PTEN-transfected HUVECs co-cultured with exosomes-miR-23a mimic was significantly reduced vs. that of oe-NC transfected HUVECs co-cultured with exosomes-miR-23a mimic (Figures 6J,K). Altogether, exosomal miR-23a could promote angiogenesis via inhibition of PTEN.

\section{DISCUSSION}

Significant implications regarding GC cell-derived miRNAs in the processes of tumorigenesis and metastasis have been previously emphasized in literature (25). miRNAs function as oncomiRs or tumor suppressive miRs in the carcinogenesis and development of upper gastrointestinal tract cancers, including GC (26). The current study placed particular emphasis on the functions of exosomal miR-23a in GC, and subsequently obtained data confirming that exosomal miR-23a induced GC progression by promoting angiogenesis via PTEN.

A key initial finding of our study revealed that miR23a was expressed at a high level in GC tissues and cells. Current studies have widely explored the role of miR-23a in human cancers. For example, miR-23a accelerates autophagy and increases survival and migration of breast cancer cells by targeting XIAP (27). A previous study concluded that miR23a up-regulates IKK alpha expression while down-regulating the expression of ST7L expression, ultimately enhancing the malignant phenotypes of epithelial ovarian cancer cells (28). The apoptosis of GC cells can be inhibited by miR-23a by down-regulating PPP2R5E (29). Additionally, miR-23a targets metallothionein $2 \mathrm{~A}$ and consequently facilitates the growth of GC cells (30). Moreover, miR-23a has been reported to promote GC cell proliferation and metastatic potential by targeting SPRY2 (31). Hence, gain-of-function of miR-23a exhibits a promotive effect on tumor progression, which may function by targeting some tumor suppressors.

In the present study, PTEN was found to be under-expressed in GC tissues, which was further verified as a target of miR23a. PTEN is a recognized tumor suppressor in various kinds of human cancers including breast cancer (32). High expression of PTEN is conducive to restrain cancer cell proliferation and decrease cell survival in colorectal cancer (33). It is reported that deletion of PTEN contributes to the occurrence and development of GC (34). PTEN can be targeted by miR-718 and miR382 and is consistently shown to inhibit the angiogenesis and progression of GC (35). Thus, miR-23a potentially played a promoting role in $\mathrm{GC}$ angiogenesis and progression by negatively regulating PTEN.

We also validated that miR-23a was enriched in cancerderived exosomes, and exosomes-delivered miR-23a facilitated angiogenesis by targeting PTEN in a co-culture system. Partially, consistent with our findings, exosomes secreted by hypoxic lung cancer cells carry miR-23a to promote angiogenesis and capillary permeability (24). Similarly, lung cancer cells-derived extracellular vesicles have been shown to enhance endothelial cell angiogenesis and radiation resistance by transferring miR23a which functionally down-regulates PTEN (36). However, this study further identified an involvement of the Akt pathway during the regulation process of miR-23a in tumor angiogenesis. The phosphatidylinositol 3-kinase (PI3K)/Akt pathway is over-activated in GC (37), and modulates a wide range of cellular processes, such as proliferation and angiogenesis (38). Additionally, restoration of PTEN expression may block angiogenesis in GC by inactivating the PI3K/Akt pathway (39). The loss of PTEN has been reported to trigger activation of the PI3K/Akt pathway, which enhances angiogenesis (40). Based on the aforementioned references and our results, it can be inferred that exosomal miR-23a may promote angiogenesis by activating the PTEN-dependent Akt pathway, which may stimulate the deterioration of cancer cells.

This study also verified the angiogenic role of exosomesdelivered miR-23a in molecular levels, as our data shown that VEGF expression was markedly increased, while TSP-1 expression was reduced by GC cell-derived exosomal miR-23a. VEGF, one of the powerful activators of angiogenesis, stimulates endothelial cell migration and proliferation abilities in existing vessels, thereby enhancing the generation and stabilization of new blood vessels $(41,42)$. TSP-1 is a multidomain glycoprotein participated in intercellular and cell to matrix interactions, and particularly related to angiogenesis and tumorigenesis (43). Furthermore, overexpression of TSP-1 is correlated with suppression of angiogenesis in human cancers such as prostate cancer (44). Those findings contribute to the conception proposed by our study that GC cell-derived exosomal miR-23a 


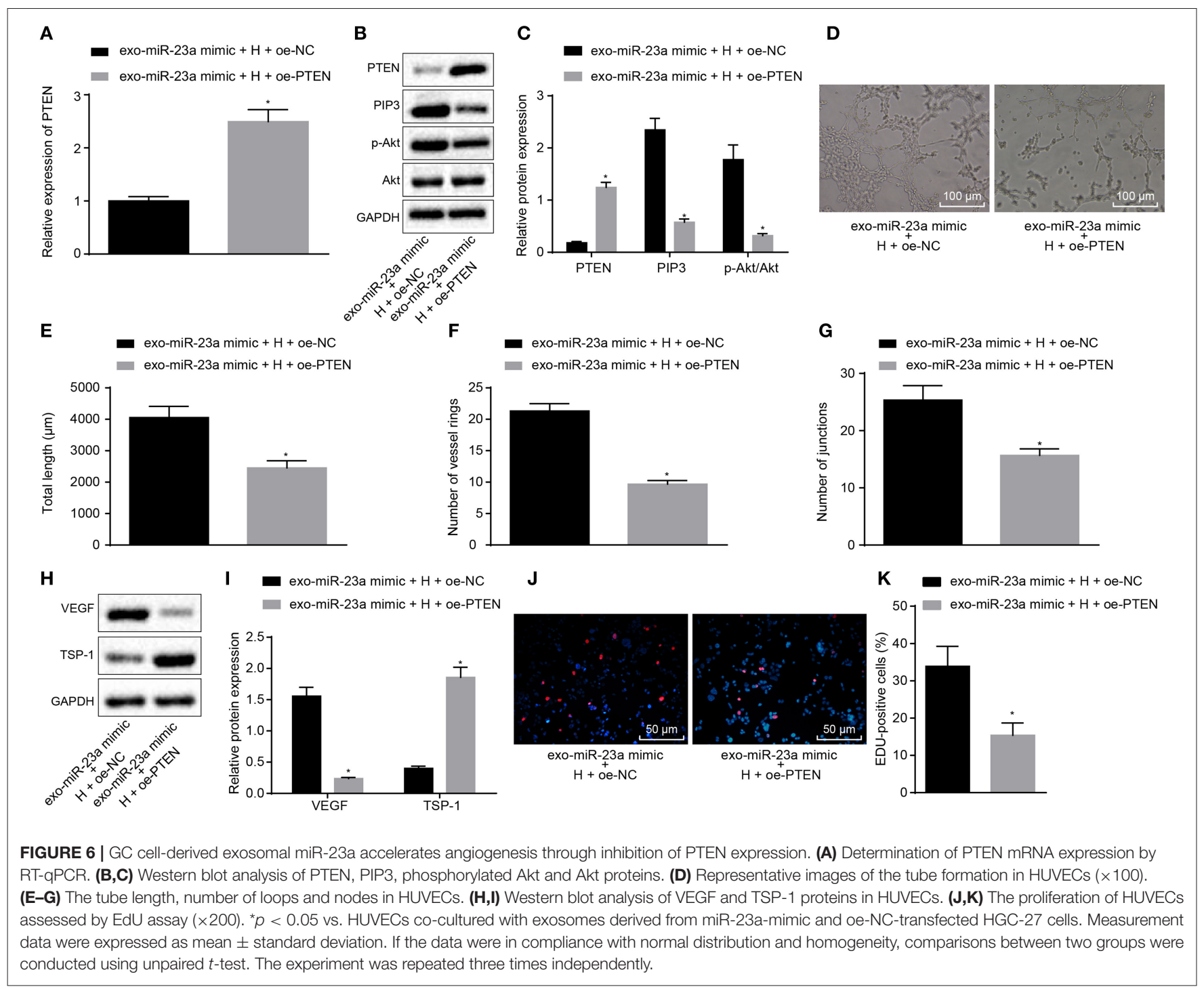

facilitates angiogenesis, as shown by increased VEGF but reduced TSP-1 levels.

Taken together, this study corroborated that miR-23a carried by exosomes inhibited PTEN to promote the development of $\mathrm{GC}$ by inducing angiogenesis. Based on the above findings, miR23a may be a promising target for GC treatment. Altogether, this study may open new avenues of research to harness the role of miR-23a in optimizing future endothelial cell-based therapies for GC. However, there were several limitations in our study. Firstly, the sample size in our study was relatively small, so further studies with larger sample sized should be conducted in the future. Secondly, due to the limited funding and technology, in vivo assays cannot be carried out temporarily. Thirdly, we only used a single dose of exosomes in our experiments. It will be necessary to perform extended time course and dose-response experiments in follow-up studies examining the translational potential of these findings. Finally, investigation into efficient, economical and effective exosome isolation techniques requires future exploration.

\section{DATA AVAILABILITY STATEMENT}

The datasets generated for this study are available on request to the corresponding author.

\section{ETHICS STATEMENT}

The studies involving human participants were reviewed and approved by the First Affiliated Hospital of China Medical University. The patients/participants provided their written informed consent to participate in this study.

\section{AUTHOR CONTRIBUTIONS}

JD, YL, JL, JMZ, and XYL designed the study. JD and YL collated the data, carried out data analyses, and produced the initial draft of the manuscript. JL, JMZ, and XYL contributed to drafting the manuscript. All authors have read and approved the final submitted manuscript. 


\section{FUNDING}

This study was supported by the China Postdoctoral Science Foundation Grant (No. 2019M661171).

\section{REFERENCES}

1. Chia NY, Tan P. Molecular classification of gastric cancer. Ann Oncol. (2016) 27:763-9. doi: 10.1093/annonc/mdw040

2. Bray F, Ferlay J, Soerjomataram I, Siegel RL, Torre LA, Jemal A. Global cancer statistics 2018: GLOBOCAN estimates of incidence and mortality worldwide for 36 cancers in 185 countries. CA Cancer J Clin. (2018) 68:394-424. doi: $10.3322 /$ caac. 21492

3. Van Cutsem E, Sagaert X, Topal B, Haustermans K, Prenen H. Gastric cancer. Lancet. (2016) 388:2654-64. doi: 10.1016/S0140-6736(16) 30354-3

4. Betzer O, Perets N, Angel A, Motiei M, Sadan T, Yadid G, et al. In vivo neuroimaging of exosomes using gold nanoparticles. ACS Nano. (2017) 11:10883-93. doi: 10.1021/acsnano.7b04495

5. Mrowczynski OD, Zacharia BE, Connor JR. Exosomes and their implications in central nervous system tumor biology. Prog Neurobiol. (2019) 172:71-83. doi: 10.1016/j.pneurobio.2018.06.006

6. Zhang H, Deng T, Liu R, Bai M, Zhou L, Wang X, et al. Exosome-delivered EGFR regulates liver microenvironment to promote gastric cancer liver metastasis. Nat Commun. (2017) 8:15016. doi: 10.1038/ncomms15016

7. Fu M, Gu J, Jiang $\mathrm{P}$, Qian $\mathrm{H}, \mathrm{Xu} \mathrm{W}$, Zhang $\mathrm{X}$. Exosomes in gastric cancer: roles, mechanisms, and applications. Mol Cancer. (2019) 18:41. doi: 10.1186/s12943-019-1001-7

8. McLean MH, El-Omar EM. Genetics of gastric cancer. Nat Rev Gastroenterol Hepatol. (2014) 11:664-74. doi: 10.1038/nrgastro.2014.143

9. Padmanabhan N, Ushijima T, Tan P. How to stomach an epigenetic insult: the gastric cancer epigenome. Nat Rev Gastroenterol Hepatol. (2017) 14:467-78. doi: $10.1038 /$ nrgastro.2017.53

10. Ueda T, Volinia S, Okumura H, Shimizu M, Taccioli C, Rossi S, et al. Relation between microRNA expression and progression and prognosis of gastric cancer: a microRNA expression analysis. Lancet Oncol. (2010) 11:136-46. doi: 10.1016/S1470-2045(09)70343-2

11. Guo H, Ingolia NT, Weissman JS, Bartel DP. Mammalian microRNAs predominantly act to decrease target mRNA levels. Nature. (2010) 466:835-40. doi: $10.1038 /$ nature 09267

12. Di Leva G, Garofalo M, Croce CM. microRNAs in cancer. Annu Rev Pathol. (2014) 9:287-314. doi: 10.1146/annurev-pathol-012513-104715

13. Song JH, Meltzer SJ. microRNAs in pathogenesis, diagnosis, and treatment of gastroesophageal cancers. Gastroenterology. (2012) 143:35-47.e2. doi: 10.1053/j.gastro.2012.05.003

14. Tanz RD, Weller TS, Chang KS. Diabetic duration and rat aortic responsiveness to histamine and nitroglycerin. Proc West Pharmacol Soc. (1988) 31:177-80.

15. Ruggieri V, Russi S, Zoppoli P, La Rocca F, Angrisano T, Falco G, et al. The role of microRNAs in the regulation of gastric cancer stem cells: a metaanalysis of the current status. J Clin Med. (2019) 8:639. doi: 10.3390/jcm80 50639

16. Ding L, Xu Y, Zhang W, Deng Y, Si M, Du Y, et al. MiR-375 frequently downregulated in gastric cancer inhibits cell proliferation by targeting JAK2. Cell Res. (2010) 20:784-93. doi: 10.1038/cr.2010.79

17. Hu X, Wang Y, Liang H, Fan Q, Zhu R, Cui J, et al. miR-23a/b promote tumor growth and suppress apoptosis by targeting PDCD4 in gastric cancer. Cell Death Dis. (2017) 8:e3059. doi: 10.1038/cddis.2017.447

18. Han Z, Zhou X, Li S, Qin Y, Chen Y, Liu H. Inhibition of miR-23a increases the sensitivity of lung cancer stem cells to erlotinib through PTEN/PI3K/Akt pathway. Oncol Rep. (2017) 38:3064-70. doi: 10.3892/or.2017.5938

19. Guo SL, Ye H, Teng Y, Wang YL, Yang G, Li XB, et al. Akt-p53-miR-365cyclin D1/cdc25A axis contributes to gastric tumorigenesis induced by PTEN deficiency. Nat Commun. (2013) 4:2544. doi: 10.1038/ncomms3544

\section{ACKNOWLEDGMENTS}

We would like to give our sincere appreciation to the reviewers for their helpful comments on this article.

20. Yang $H$, Zhang $H$, Ge S, Ning $T$, Bai $M$, Li J, et al. Exosomederived miR-130a activates angiogenesis in gastric cancer by targeting C-MYB in vascular endothelial cells. Mol Ther. (2018) 26:2466-75. doi: 10.1016/j.ymthe.2018.07.023

21. Ye ZL, Huang Y, Li LF, Zhu HL, Gao HX, Liu H, et al. Argonaute 2 promotes angiogenesis via the PTEN/VEGF signaling pathway in human hepatocellular carcinoma. Acta Pharmacol Sin. (2015) 36:1237-45. doi: 10.1038/aps.2015.18

22. Bao L, You B, Shi S, Shan Y, Zhang Q, Yue H, et al. Metastasis-associated miR23a from nasopharyngeal carcinoma-derived exosomes mediates angiogenesis by repressing a novel target gene TSGA10. Oncogene. (2018) 37:2873-89. doi: 10.1038/s41388-018-0183-6

23. Sruthi TV, Edatt L, Raji GR, Kunhiraman H, Shankar SS, Shankar V, et al. Horizontal transfer of miR-23a from hypoxic tumor cell colonies can induce angiogenesis. J Cell Physiol. (2018) 233:3498-514. doi: 10.1002/jcp.26202

24. Hsu YL, Hung JY, Chang WA, Lin YS, Pan YC, Tsai PH, et al. Hypoxic lung cancer-secreted exosomal miR-23a increased angiogenesis and vascular permeability by targeting prolyl hydroxylase and tight junction protein ZO-1. Oncogene. (2017) 36:4929-42. doi: 10.1038/onc.2017.105

25. Liu HS, Xiao HS. microRNAs as potential biomarkers for gastric cancer. World J Gastroenterol. (2014) 20:12007-17. doi: 10.3748/wjg.v20.i34.12007

26. Song S, Ajani JA. The role of microRNAs in cancers of the upper gastrointestinal tract. Nat Rev Gastroenterol Hepatol. (2013) 10:109-18. doi: $10.1038 /$ nrgastro.2012.210

27. Chen $\mathrm{P}$, He YH, Huang $\mathrm{X}$, Tao SQ, Wang $\mathrm{XN}$, Yan $H$, et al. MiR23a modulates X-linked inhibitor of apoptosis-mediated autophagy in human luminal breast cancer cell lines. Oncotarget. (2017) 8:80709-21. doi: 10.18632/oncotarget.21080

28. Yang Z, Wang XL, Bai R, Liu WY, Li X, Liu M, et al. miR-23a promotes IKKalpha expression but suppresses ST7L expression to contribute to the malignancy of epithelial ovarian cancer cells. Br J Cancer. (2016) 115:731-40. doi: 10.1038/bjc.2016.244

29. Liu X, Liu Q, Fan Y, Wang S, Liu X, Zhu L, et al. Downregulation of PPP2R5E expression by miR-23a suppresses apoptosis to facilitate the growth of gastric cancer cells. FEBS Lett. (2014) 588:3160-9. doi: 10.1016/j.febslet.2014.05.068

30. An J, Pan Y, Yan Z, Li W, Cui J, Yuan J, et al. MiR-23a in amplified 19p13.13 loci targets metallothionein $2 \mathrm{~A}$ and promotes growth in gastric cancer cells. $J$ Cell Biochem. (2013) 114:2160-9. doi: 10.1002/jcb.24565

31. Li Y, Chen H, She P, Chen T, Chen L, Yuan J, et al. microRNA-23a promotes cell growth and metastasis in gastric cancer via targeting SPRY2mediated ERK signaling. Oncol Lett. (2018) 15:8433-41. doi: 10.3892/ol.2018. 8374

32. Kazim Z, Wahabi K, Perwez A, Lal P, Rizvi MA. PTEN genetic and epigenetic alterations define distinct subgroups in North Indian Breast Cancer Patients. Asian Pac J Cancer Prev. (2019) 20:269-76. doi: 10.31557/APJCP.2019.20.1.269

33. Yang R, Cai TT, Wu XJ, Liu YN, He J, Zhang XS, et al. Tumour YAP1 and PTEN expression correlates with tumour-associated myeloid suppressor cell expansion and reduced survival in colorectal cancer. Immunology. (2018) 155:263-72. doi: 10.1111/imm.12949

34. Kim SJ, Lee HW, Baek JH, Cho YH, Kang HG, Jeong JS, et al. Activation of nuclear PTEN by inhibition of Notch signaling induces G2/M cell cycle arrest in gastric cancer. Oncogene. (2016) 35:251-60. doi: 10.1038/onc. 2015.80

35. Liu S, Tian Y, Zhu C, Yang X, Sun Q. High miR-718 suppresses phosphatase and tensin homolog (PTEN) expression and correlates to unfavorable prognosis in gastric cancer. Med Sci Monit. (2018) 24:5840-50. doi: 10.12659/MSM.909527

36. Zheng Y, Liu L, Chen C, Ming P, Huang Q, Li C, et al. The extracellular vesicles secreted by lung cancer cells in radiation therapy promote 
endothelial cell angiogenesis by transferring miR-23a. PeerJ. (2017) 5:e3627. doi: $10.7717 /$ peerj.3627

37. Tapia O, Riquelme I, Leal P, Sandoval A, Aedo S, Weber H, et al. The PI3K/AKT/mTOR pathway is activated in gastric cancer with potential prognostic and predictive significance. Virchows Arch. (2014) 465:25-33. doi: 10.1007/s00428-014-1588-4

38. Lin FC, Liu YP, Lai CH, Shan YS, Cheng HC, Hsu PI, et al. RUNX3-mediated transcriptional inhibition of Akt suppresses tumorigenesis of human gastric cancer cells. Oncogene. (2012) 31:4302-16. doi: 10.1038/onc.2011.596

39. Wu ZH, Lin C, Liu CC, Jiang WW, Huang MZ, Liu X, et al. MiR-616-3p promotes angiogenesis and EMT in gastric cancer via the PTEN/AKT/mTOR pathway. Biochem Biophys Res Commun. (2018) 501:1068-73. doi: 10.1016/j.bbrc.2018.05.109

40. Xue L, Huang J, Zhang T, Wang X, Fu J, Geng Z, et al. PTEN inhibition enhances angiogenesis in an in vitro model of ischemic injury by promoting Akt phosphorylation and subsequent hypoxia inducible factor-1alpha upregulation. Metab Brain Dis. (2018) 33:1679-88. doi: 10.1007/s11011-018-0276-5

41. Dong G, Lin XH, Liu HH, Gao DM, Cui JF, Ren ZG, et al. Intermittent hypoxia alleviates increased VEGF and pro-angiogenic potential in liver cancer cells. Oncol Lett. (2019) 18:1831-39. doi: 10.3892/ol.2019. 10486
42. Laddha AP, Kulkarni YA. VEGF and FGF-2: promising targets for the treatment of respiratory disorders. Respir Med. (2019) 156:33-46. doi: 10.1016/j.rmed.2019.08.003

43. Ren J, Gu C, Yang Y, Xue J, Sun Y, Jian F, et al. TSP-1 is downregulated and inversely correlates with miR-449c expression in Cushing's disease. J Cell Mol Med. (2019) 23:4097-110. doi: 10.1111/jcmm.14297

44. Yang F, Jiang X, Song L, Wang H, Mei Z, Xu Z, et al. Quercetin inhibits angiogenesis through thrombospondin-1 upregulation to antagonize human prostate cancer PC-3 cell growth in vitro and in vivo. Oncol Rep. (2016) 35:1602-10. doi: $10.3892 /$ or.2015.4481

Conflict of Interest: The authors declare that the research was conducted in the absence of any commercial or financial relationships that could be construed as a potential conflict of interest.

Copyright $\odot 2020 \mathrm{Du}$, Liang, Li, Zhao and Lin. This is an open-access article distributed under the terms of the Creative Commons Attribution License (CC BY). The use, distribution or reproduction in other forums is permitted, provided the original author(s) and the copyright owner(s) are credited and that the original publication in this journal is cited, in accordance with accepted academic practice. No use, distribution or reproduction is permitted which does not comply with these terms. 